\title{
APLICAÇÃO DO ALGORITMO S-SEBI NA OBTENÇÃO DA EVAPOTRANSPIRAÇÃO DIÁRIA EM CONDIÇÕES ÁRIDAS
}

\author{
CARLOS ANTONIO COSTA DOS SANTOS
}

\author{
Unidade Acadêmica de Ciências Atmosféricas, Universidade Federal de Campina Grande (UFCG), Campina \\ Grande, PB \\ carlostorm@gmail.com e carlos@dca.ufcg.edu.br
}

Recebido Agosto 2009 - Aceito Janeiro 2010

\begin{abstract}
RESUMO
O principal objetivo desse estudo foi determinar a evapotranspiração real diária $(E T r)$ da vegetação tamarisk através de técnicas micrometeorológicas e de sensoriamento remoto, e validar os resultados da ETr estimados pelo sensoriamento remoto. Foram utilizados dados micrometeorológicos provinientes do método da razão de Bowen, além do algoritmo S-SEBI aplicado a imagens do Landsat 5 - TM, na obtenção da $E T r$ diária da vegetação tamarisk do Baixo Rio Colorado, CA/EUA. Na obtenção da evapotranspiração de referência $\left(E T_{0}\right)$ foram utilizados os dados de uma estação meteorológica e o método utilizado foi o de FAO/Penman-Monteith. Foram observados que as estimativas da ETr pelo algoritmo S-SEBI são similares aos valores medidos na torre micrometeorológica, assim como, que a identificação da dinâmica da vegetação através da distribuição espacial da evapotranspiração, evidencia a aplicabilidade do método na obtenção da evapotranspiração real diária.
\end{abstract}

Palavras-chave: Balanço de energia, micrometeorologia, Landsat 5 - TM, tamarisk

\begin{abstract}
APPLICATION OF THE S-SEBI ALGORITHM TO OBTAIN THE DAILY EVAPOTRANSPIRATION IN ARID CONDITIONS

The main objective of this study was to determine the daily actual evapotranspiration (ETr) of the tamarisk vegetation through micrometeorological and remote sensing techniques and to validate the results of ETr obtained by remote sensing. Micrometeorological data to obtain the daily ETr of the tamarisk vegetation in the Lower Colorado River, CA/USA provided by the Bowen ratio method, besides of the S-SEBI algorithm applied to TM Landsat-5 images were used. For obtaining the reference evapotranspiration (ET0) the weather station data were used and the FAO/Penman-Monteith method was applied. It was observed that the estimates of ETr by S-SEBI algorithm are similar to the measured values by the Bowen ratio method, as well as, that the identification of the vegetation dynamics through the spatial distribution of the evapotranspiration evidences the method applicability in obtaining the daily actual evapotranspiration.
\end{abstract}

Keywords: Energy balance, micrometeorology, TM Landsat 5, tamarisk

\section{1 - INTRODUÇÃO}

A água é o recurso natural mais limitante em ambientes áridos e semi-áridos. Vegetação nativa, agricultura irrigada e desenvolvimento social, dependem da produção e conservação da água de rios e aquíferos. A obtenção da evapotranspiração (ET) (fenômeno físico que transfere grande volume de água para a atmosfera através da evaporação do solo e do processo da transpiração vegetativa) da vegetação ribeirinha é um importante componente do balanço hídrico para bacias hidrográficas, localizadas nas regiões áridas e semi-áridas. Os principais rios da região ocidental dos Estados Unidos da América (EUA), apresentam vegetações invasoras que causam prejuízos econômicos estimados em dezenas de bilhões de dólares por ano, 
causando alterações nos ecossistemas (Pimentel et al., 2001) e o consumo hídrico dessas vegetações necessita ser melhor estudado (Mack et al., 2000). Assim, a aplicação de técnicas de sensoriamento remoto orbital na obtenção da ET constitui um elemento indispensável.

A espécie conhecida como tamarisk (Tamarix spp.) é uma vegetação arbustiva que invadiu a região oeste dos EUA, no início do século passado, e tem sido alvo de muitos esforços na tentativa de controlá-la, assim como, de restauração ambiental, começando na década de 1960 (Weeks et al., 1987). Atualmente, programas direcionam milhões de dólares por ano na tentativa de controlar a rápida expansão do tamarisk e, com isso, aumentar a produtividade da água e a saúde do ecossistema. Tamarisk é comumente conhecida por ter efeitos ecológicos e econômicos negativos, como a diminuição das correntes dos rios, resultante da alta taxa de $E T$, deslocamento da vegetação nativa, aumento da salinização, entre outros. No entanto, os benefícios da tamarisk estão na sua alta capacidade de controlar erosão, no fato de se tornar habitat para diferentes espécies de pássaros (van Ripper III et al., 2008), incluindo locais com alto teor de salinidade ou seco, onde a vegetação nativa não se desenvolve, como por exemplo, no Baixo Rio Colorado e no Rio Pecos (Shafroth et al., 2005). Logo, quantificar o consumo hídrico sobre grandes áreas é importante para o manejo adequado, planejamento e gestão dos recursos hídricos. As diferenças entre a ET real e potencial obtidas com alta resolução espacial são de interesses para os recursos hídricos e constituem um indicador do déficit hídrico.

Com isso, várias técnicas têm sido desenvolvidas com a finalidade de obter precisamente a $E T$, entre elas estão: Razão de Bowen (Bowen, 1926) e correlações turbulentas (Swinbank, 1951), assim como, de estimar espacialmente a ET utilizando sensoriamento remoto orbital, a exemplo do S-SEBI (Simplified Surface Energy Balance Index), proposto por Roerink et al. (2000). O S-SEBI utiliza uma metodologia simples para a obtenção do balanço de energia e, por conseqüência, da $E T$, com base apenas no contraste da fração evaporativa entre áreas secas e úmidas. O S-SEBI foi validado na Itália, na Espanha, na Holanda e na Península Ibérica (Sobrino et al., 2007). O fato de este algoritmo necessitar apenas das informações contidas na imagem para estimar o balanço de energia o torna uma ferramenta promissora para a estimativa espacial da $E T$.

O principal objetivo desse estudo é determinar a evapotranspiração real diária $(E T r)$ da vegetação tamarisk utilizando o método da razão de Bowen e o algoritmo S-SEBI, através de imagens de alta resolução espacial (Landsat 5-TM), e validar os resultados da ETr estimados pelo algoritmo com os medidos na torre micrometeorológica.

\section{MATERIAL E MÉTODOS}

\section{1 Área de Estudo}

A área de estudo foi uma reserva florestal que serve de refúgio para animais e pássaros silvestres, conhecida por Cibola National Wildlife Refuge (CNWR), situada a $33^{\circ} 16^{\prime} \mathrm{N}, 114^{\circ} 41^{\prime}$ $\mathrm{W}$ e $70 \mathrm{~m}$ acima do nível do mar. A referida área fica localizada no Baixo Rio Colorado, no Estado da Califórnia, EUA, conforme ilustrado na Figura 1. O CNWR foi criado em 1964 com o objetivo de restaurar e preservar o histórico habitat de aves migratórias e outros animais silvestres. A região é cercada pelo Rio Colorado e pelo deserto de Sonora, apresentando clima típico de deserto com umidade relativa extremamente baixa e temperaturas extremamente altas no verão. Suas temperaturas

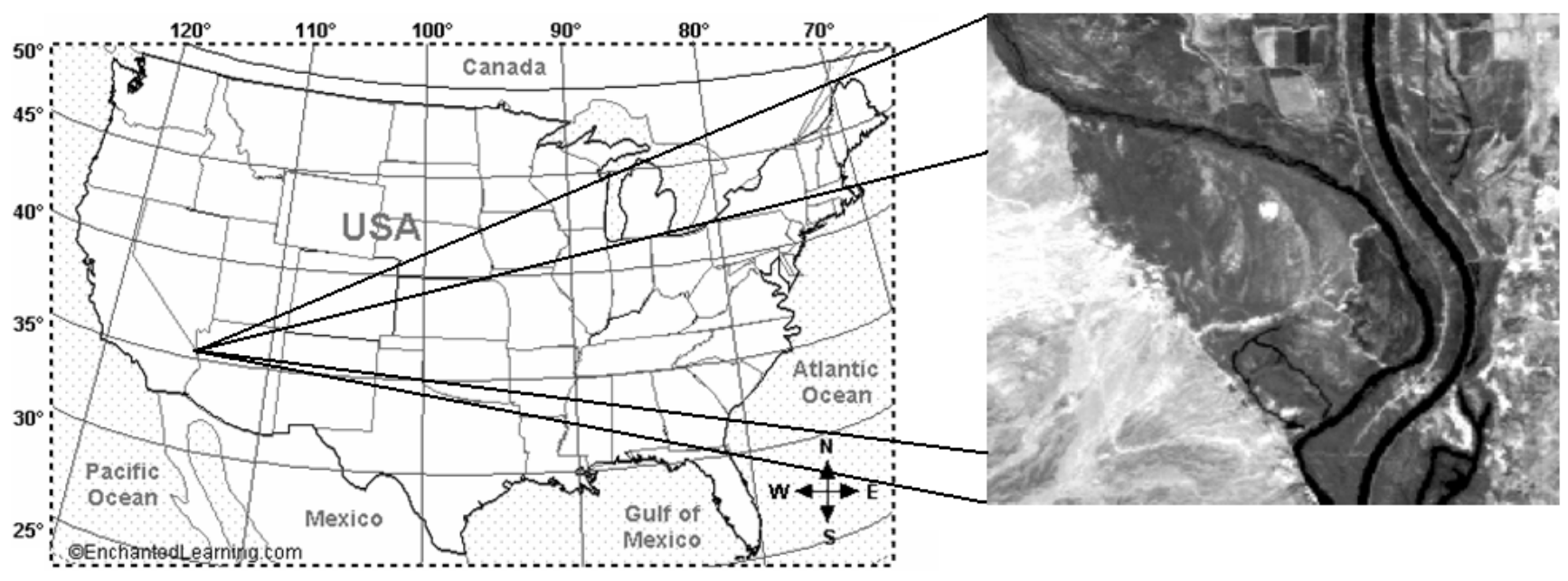

Figura 1 - Mapa da área de estudo na reserva CNWR no Baixo Rio Colorado juntamente com as áreas irrigadas. 
média, máxima e mínima anuais são de $22,2^{\circ} \mathrm{C}, 31,2^{\circ} \mathrm{C}$ e 13,2 ${ }^{\circ} \mathrm{C}$, respectivamente. A precipitação média anual da região é de $97,3 \mathrm{~mm}$.

\subsection{Metodologia}

\subsubsection{Método da Razão de Bowen}

Foram utilizados nesse estudo dados micrometeorológicos provinientes do método da razão de Bowen, além do algoritmo S-SEBI aplicado a imagens de alta resolução espacial $(30 \mathrm{~m})$ do Landsat 5 - TM, para a obtenção e comparação da $E \operatorname{Tr}$ diária da vegetação tamarisk.

Foi instalada uma torre micrometeorológica sobre a área de maior densidade da vegetação tamarisk, dentro da CNWR. Para a obtenção da $E T_{0}$ foram utilizados os dados de uma estação meteorológica do Sistema de Informação e Monitoramento da Irrigação na California (CIMIS, em inglês) instalada próxima a área de estudo, com localização $33^{\circ} 33^{\prime} 24^{\prime \prime} \mathrm{N}, 114^{\circ} 39^{\prime} 59^{\prime \prime} \mathrm{W}$ e 84 de altitude. A superfície de referência foi grama e o método utilizado foi o de Penman-Monteith (Allen et al., 1998). Todos os instrumentos utilizados na estação meteorológica estão descritos na Tabela 1.

A partição da energia na superfície entre fluxo de calor sensível e latente é usualmente obtida através do método do balanço de energia pela razão de Bowen (BERB) (Perez et al., 2008). Nesse estudo foi utilizado o sistema do balanço de energia CR10-3C confeccionado pela Radiation and Energy Balance Systems, Inc. (REBS), com os sensores para a medição de temperatura e umidade instalados, em geral, a 2 e 3 metros acima do dossel da vegetação tamarisk, em CNWR. Os componentes do BERB foram obtidos a cada 30 minutos. O método do BERB é baseado na teoria de que os fluxos unidimensionais de calor sensível e latente podem ser descritos em termos das relações fluxos-gradiente (Tanner et al, 1987; Tanner, 1988):

$$
H=\rho c_{p} K_{h}(\Delta T / \Delta z)
$$

$$
L E=\left(\lambda \rho \varepsilon K_{w} / P\right)(\Delta e / \Delta z)
$$

onde $H$ é o fluxo de calor sensível da superfície $\left(\mathrm{Wm}^{-2}\right), L E$ é o fluxo de calor latente da superfície $\left(\mathrm{Wm}^{-2}\right)$, $\rho$ é a densidade do ar $\left(\mathrm{kgm}^{-3}\right), c_{p}$ é o calor específico do ar à pressão constante $\left(\mathrm{Jkg}^{-1}{ }^{\circ} \mathrm{C}^{-1}\right), T$ é a temperatura do ar $\left({ }^{\circ} \mathrm{C}\right), z$ é a altura em que são obtidas as medidas $(\mathrm{m}), \lambda$ é o calor latente de vaporização $\left(\mathrm{Jkg}^{-1}\right), \varepsilon$ é a razão do peso molecular da água pelo do ar seco $(0,622), P$ é a pressão atmosférica $(\mathrm{kPa}), e$ é a pressão de vapor $(\mathrm{kPa}), K_{h}$ é o coeficiente de difusão turbulenta para o calor $\left(\mathrm{m}^{2} \mathrm{~s}^{-1}\right)$ e $K_{w}$ é o coeficiente de difusão turbulenta para o vapor de água $\left(\mathrm{m}^{2} \mathrm{~s}^{-1}\right)$.

Bowen (1926) expressou a razão de Bowen ( $\beta$ ) como:

$$
\beta=H / L E
$$

Substituindo as Equações 1 e 2 na Equação 3, e assumindo $K_{h}=K_{w}$ (Verma et al., 1978), $\beta$ pode ser obtido por (Bowen, 1926):

$$
\beta=\gamma(\Delta T / \Delta e)
$$

onde $\gamma$ é o fator psicrométrica $\left(\mathrm{kPa}^{\circ} \mathrm{C}^{-1}\right), \Delta T$ e $\Delta e$ são obtidos pelas medidas da temperatura do ar e pressão de vapor ou ponto de orvalho para duas alturas sobre o topo do dossel, dentro da camada limite planetária (Payero et al., 2003).

A equação unidimensional do balanço de energia na superfície é dada a seguir:

$$
L E=R_{n}-G-H
$$

onde $R_{n}$ é o saldo de radiação, $G$ é o fluxo de calor no solo, $H$ é o fluxo de calor sensível e $L E$ o fluxo de calor latente, todos espressos em $\mathrm{Wm}^{-2}$. Combinando as Equações 4 e 5, resulta

Tabela 1 - Instrumentação utilizada na estação meteorológica instalada nas proximidades de CNWR, Baixo Rio Colorado, CA, EUA

\begin{tabular}{lcc}
\hline \multicolumn{1}{c}{ Medida } & Instrumento & Altura (m) \\
\hline Radiação solar total & $\begin{array}{c}\text { LI200S (Li-Cor) } \\
\text { (Piranômetro) }\end{array}$ & 2,0 \\
Temperatura do solo & 107b & $-0,15$ \\
Temperatura e umidade relative do ar & (Fenwal/Campbell Scientific Inc.) & 1,5 \\
Direção e velocidade do vento & HMP35 & 2,0 \\
Precipitação & (Vaisala/Campbell Scientific, Inc.) & 1,0 \\
& $\begin{array}{c}\text { 024A/014A } \\
\text { (Met-One) }\end{array}$ \\
\hline
\end{tabular}


a seguinte equação para calcular $L E$ pelos dados da razão de Bowen (Bowen, 1926):

$$
L E=\left(R_{n}-G\right) /(1+\beta)
$$

O fluxo de calor no solo foi obtido através de três fluxímetros, modelo HFT3.1, instalados a $8 \mathrm{~cm}$ de profundidade. O saldo de radiação foi medido com um saldo radiômetro modelo THRDS7.1 instalados a $2 \mathrm{~m}$ acima do dossel. Foi utilizado um piranômetro do modelo PDS7.1 para medir a radiação solar incidente. Na obtenção da umidade e temperatura do solo, foram utilizadas sensores dos modelos SMP1 e STP, respectivamente. Um sensor do modelo Met One 034-B foi utilizado para a obtenção da velocidade e direção do vento. Todos os instrumentos citados acima foram confeccionados pela Radiation and Energy Balance Systems (REBS), Seatle, WA.

Foram aplicados filtros para a identificação de dados errôneos, seguindo a metodologia proposta por Payero et al. (2003), tais como: a exclusão dos dados, quando $-1,25<$ $\beta<-0,75$; a exclusão dos dados, quando a razão de Bowen apresentou resultados imprecisos com valores irracionais e a exclusão dos dados noturnos $\left(R_{n}<0 \mathrm{~W} \mathrm{~m}^{-2}\right)$, ou seja, utilizou-se para a obtenção da ETr diária, somente os valores diurnos do LE. Estudos como os de Heilman et al. (1989) e Payero et al. (2003) afirmam que a razão entre o fetch e a altura dos instrumentos é de 100:1. Nesse estudo, se encontrou fetch suficiente para a aplicação da técnica.

\subsubsection{Algoritmo S-SEBI}

Foram utilizadas nesse estudo imagens do Mapeador Temático a bordo do Landsat 5. A Tabela 2 apresenta as sete imagens obtidas durante o período de estudo, com o "path" 38 e "row" 37. As imagens foram utilizadas, juntamente com os dados provinientes da estação meteorológica, na obtenção da ETr diária.

O cômputo das componentes do saldo de radiação $\left(R_{n}\right)$ e fluxo de calor no solo $(G)$ foi obtido através das Equações 7 e 8 , apresentadas a seguir:

$$
R_{n}=R_{S \downarrow}-\alpha \cdot R_{S \downarrow}+R_{L \downarrow}-R_{L \uparrow}-\left(1-\varepsilon_{0}\right) R_{L \downarrow}
$$

onde $R_{S \downarrow}$ é a radiação de onda curta ou solar incidente $\left(\mathrm{Wm}^{-2}\right)$, o termo $\alpha R_{s \downarrow}$ é a porção da radiação de onda curta refletida pela superfície $\left(\mathrm{Wm}^{-2}\right), \alpha$ é o albedo da superfície, $R_{L \downarrow}$ é a radiação de onda longa emitida pela atmosfera na direção da superfície $\left(\mathrm{Wm}^{-2}\right), R_{L \uparrow}$ é a radiação de onda longa emitida pela superfície $\left(\mathrm{Wm}^{-2}\right)$; o termo $\left(1-\varepsilon_{0}\right) R_{L \downarrow}$ corresponde a radiação de onda longa refletida e $\varepsilon_{0}$ é emissividade da superfície. As etapas computacionais para a obtenção do balanço de radiação, através do algoritmo S-SEBI estão apresentadas no fluxograma da Figura 2.

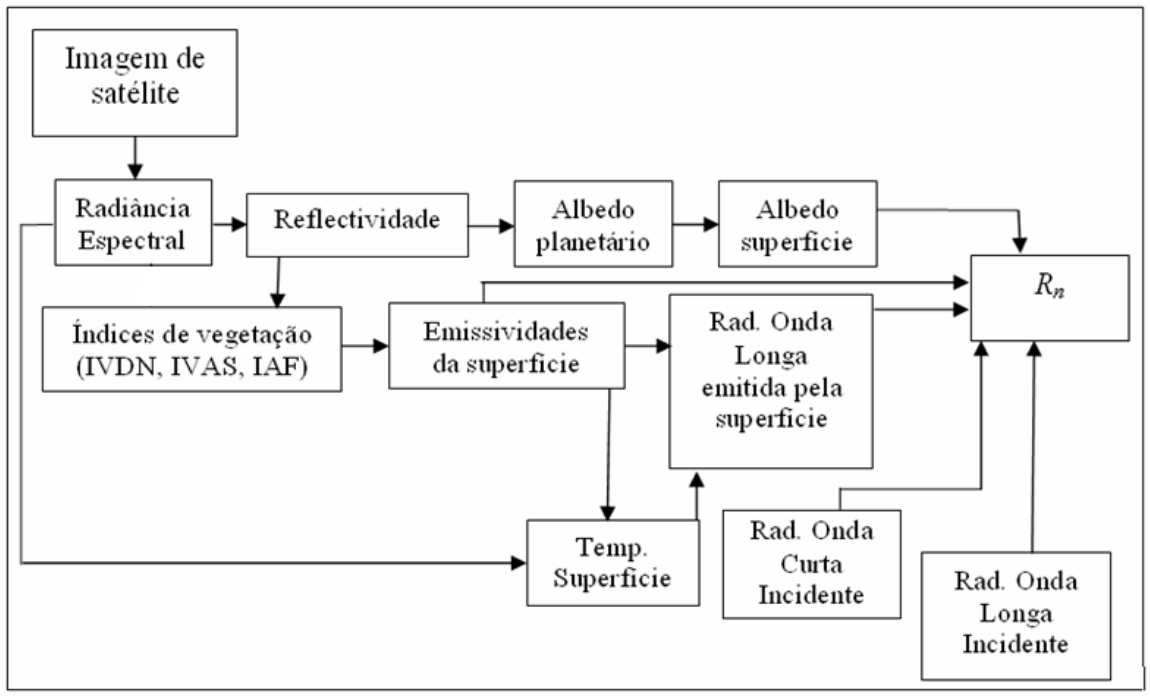

Figura 2 - Fluxograma das etapas do processamento para obtenção do balanço de radiação à superfície $(\mathrm{Rn})$. 
Tabela 2 - Data da aquisição e hora da passagem do satélite para as imagens do sensor TM do Landsat 5 (path/row = 216/64) disponíveis para a área de estudo (CNWR), em 2007da superfície e a reflectância da superfície.

\begin{tabular}{cc}
\hline Data da aquisição & Hora da passagem \\
\hline $08-05-2007$ & $11: 04$ \\
$24-05-2007$ & $11: 04$ \\
$09-06-2007$ & $11: 04$ \\
$11-07-2007$ & $11: 03$ \\
$12-08-2007$ & $11: 03$ \\
$28-08-2007$ & $11: 03$ \\
$13-09-2007$ & $11: 03$ \\
\hline
\end{tabular}

O valor de $G$ foi computado segundo a equação empírica desenvolvida por Bastiaanssen (2000):

$$
G=\left[\frac{T_{s}}{\alpha}\left(0,0038 \alpha+0,0074 \alpha^{2}\right)\left(1-0,98 I V D N^{4}\right)\right] R_{n}
$$

onde Ts é a temperatura da superfície $\left({ }^{\circ} \mathrm{C}\right), \alpha$ é o albedo da superfície, IVDN é o índice de vegetação da diferença normalizada e $\mathrm{R}_{\mathrm{n}}$ é o saldo de radiação.

A principal diferença entre o S-SEBI e outros algoritmos, a exemplo do SEBAL (Bastiaanssen et al., 1998a,b), está no cálculo dos fluxos de $H$ e $L E$. No modelo S-SEBI os fluxos não são calculados como parâmetros separados, mas através da fração evaporativa $(\Lambda)$ (Sobrino et al., 2005).

O cálculo da fração evaporativa para cada pixel com reflectância $\alpha_{0}$ e temperatura $T_{0}$ foi obtido da seguinte forma (Roerink et al., 2000; Sobrino et al., 2007):
1) determinação da reflectância dependente da temperatura $T_{L E}$, onde $L E_{\max }\left(\alpha_{0}\right)=R_{n}-G$ e $H=0$;

2) determinação da reflectância dependente da temperatura $T_{H}$, onde $H_{\max }\left(\alpha_{0}\right)=R_{n}-G$ e $L E=0$.

A fração evaporativa $(\Lambda)$ para um pixel particular foi calculada como a razão dada por:

$$
\Lambda=\frac{T_{H}-T_{0}}{T_{H}-T_{L E}}
$$

A Figura 3 mostra as características espaciais da reflectância da superfície e da temperatura da superfície. Na distribuição espacial foi possível reconhecer o limite inferior, onde o fluxo de calor latente foi assumido ser máximo $L E_{\max }\left(\alpha_{0}\right)$, e o limite superior localizado onde o fluxo de calor sensível foi máximo $H_{\max }\left(\alpha_{0}\right)$ (Sobrino et al., 2005; Gómez et al., 2005; Santos e Silva, 2008). A equação linear, que representa o limite máximo e mínimo, pode ser escrita como:

$$
\begin{gathered}
T_{H}=a_{H}+b_{H} \alpha_{0} \\
\mathrm{e} \\
T_{L E}=a_{L E}+b_{L E} \alpha_{0}
\end{gathered}
$$

As variáveis de regressão $a$ e $b$ são locais e temporais. Substituindo a relação observada por $T_{H}$ e $T_{L E}$, a fração evaporativa pode ser escrita como:

$$
\Lambda=\frac{a_{H}+b_{H} \alpha_{0}-T_{0}}{a_{H}-a_{L E}+\left(b_{H}-b_{L E}\right) \alpha_{0}}
$$

Umavezdeterminadaa $\Lambda$, os fluxos de calorsensívele latente foram calculados conforme descrito abaixo (Santos e Silva, 2008):

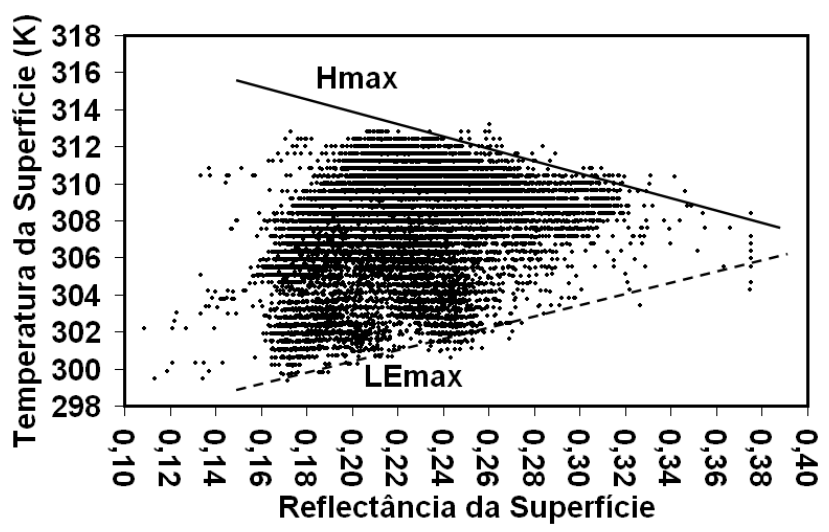

Figura 3 - Representação esquemática da relação entre a temperatura da superfície e a reflectância da superfície. 


$$
H=(1-\Lambda)\left(R_{n}-G\right)
$$

$$
L E=\Lambda\left(R_{n}-G\right)
$$

\subsubsection{Estimativa da Evapotranspiração Real Diária $(E T r)$}

A evapotranspiração real diária foi calculada a partir da evapotranspiração real horária $\left(E T_{h}\right)\left(\mathrm{mm} \mathrm{h}^{-1}\right)$, que foi obtida com base na densidade de fluxo de calor latente, de acordo com a Equação 15, onde $L$ é o calor latente de vaporização da água $\left(L=2,45 \times 10^{6} \mathrm{~J} \mathrm{~kg}^{-1}\right), 3600$ é o fator de conversão de valores instantâneo para valores horários.

$$
E T_{h}=3600 L E / L
$$

O passo seguinte foi a obtenção da fração de evapotranspiração de referência horária, $F E T_{0} h$, que foi obtida pela razão entre a $E T_{h}$ e a evapotranspiração de referência horária, $E T_{0 \_}$, calculada pelo método da FAO Penman-Monteith (Allen et al., 1998), com dados da estação meteorológica instalada na área estudada:

$$
F E T_{0_{-} h}=E T_{h} / E T_{0_{-} h}
$$

Considerando que o valor da $F E T_{0 \_}=F E T_{0 \_24}$, pois de acordo com Trezza (2002) o comportamento de $F E T_{0}{ }_{h}$ é relativamente constante em todo o período diurno. Logo, a $E T_{r}$ diária foi dada pela equação seguinte:

$$
E T r=F E T_{0 \_h} E T_{0 \_24}\left(\mathrm{~mm} \mathrm{dia}^{-1}\right)
$$

em que $F E T_{0 \_}$é a fração de evapotranspiração real diária e $E T_{0 \_24}$ é a evapotranspiração de referência diária, calculada com metodologia similar à da $E T_{0} h$.

\section{RESULTADOS E DISCUSSÃO}

\subsection{Condições Meteorológicas na Área de Estudo}

As médias diárias das variáveis meteorológicas durante o período estudado, na estação meteorológica instalada próxima a área experimental em CNWR, estão apresentadas na Figura 4. A velocidade do vento alcançou valores superiores a 2,0 $\mathrm{ms}^{-1}$ para a maioria dos dias estudados. Entretanto, em alguns dias foram identificados valores médios diários superiores a 5,0 $\mathrm{ms}^{-1}$. $\mathrm{Na}$ Figura $4 \mathrm{a}$, pode ser evidenciado que a maior variação na velocidade média diária do vento ocorre no período mais frio do ano. Durante este período, os valores variaram de $1,0 \mathrm{~ms}^{-1}$ a valores superiores a $5,0 \mathrm{~ms}^{-1}$.

O comportamento temporal da umidade relativa está apresentado na Figura 4b, mostrando que os valores máximos variaram entre $30 \%$ e $100 \%$, os valores médios entre $20 \%$ e $90 \%$, e os mínimos entre valores inferiores a $10 \%$ e $80 \%$. A temperatura do ar (Figura 4c) alcançou valores máximos acima de $40^{\circ} \mathrm{C}$, valores médios entre $3^{\circ} \mathrm{C}$ e $37^{\circ} \mathrm{C}$, e mínimos abaixo de $0^{\circ} \mathrm{C}$. Pode se evidenciar, no comportamento da temperatura do ar, o clima desértico na região estudada, com altos valores durante o dia e baixos valores à noite.

A distribuição anual das temperaturas do ar e do solo para a torre micrometeorológica instalada na área de estudo está apresentada na Figura 5. Pode ser vista, na referida figura, a distribuição das temperaturas na parte mais densa da vegetação tamarisk, dentro da CNWR, em 2007. Pode ser evidenciado que a temperatura do solo se mantém, durante todo o ano, bastante inferior à temperatura do ar, devido ao fato da vegetação ser densa, impedindo a incidência direta da radiação solar no solo.

\subsection{Evapotranspiração Real da Vegetação Tamarisk}

Na Tabela 3 estão apresentados os valores da ETr média diária para o período de $1^{\circ}$ de março a 31 de outubro, período em que a temperatura do ar é superior a $0^{\circ} \mathrm{C}$ e que as plantas estão

Tabela 3 - Média sazonal (01/março a 31/outubro) e valores totais anuais da ET0 e da ETr da vegetação tamarisk obtida para a torre instalada em CNWR em 2007 juntamente com a precipitação total.

\begin{tabular}{lccc}
\hline \multicolumn{1}{c}{ Torre } & $\begin{array}{c}\text { ETr média } \\
(\mathbf{m m} / \mathbf{d i a})\end{array}$ & $\begin{array}{c}\text { ETr } \\
(\mathbf{m m} / \mathbf{a n o})\end{array}$ & $\begin{array}{c}\text { Precipitação Total } \\
(\mathbf{m m})\end{array}$ \\
\hline $\mathrm{ET}_{0}$ (grama) & 4,8 & 1734,2 & 37,3 \\
Tamarisk (Razão de Bowen) & 3,2 & 1096,2 & \\
\hline
\end{tabular}


em desenvolvimento, para o ano de 2007, da vegetação tamarisk juntamente com a $E T_{0}$ sobre grama. É possível identificar que a ETr da vegetação tamarisk apresentou um valor médio de 3,2 $\mathrm{mm} \mathrm{dia}{ }^{-1}$. Este resultado está em concordância com os estudos desenvolvidos por Nagler et al. (2008) para a região estudada utilizando sensoriamento remoto e torres micrometeorológicas.

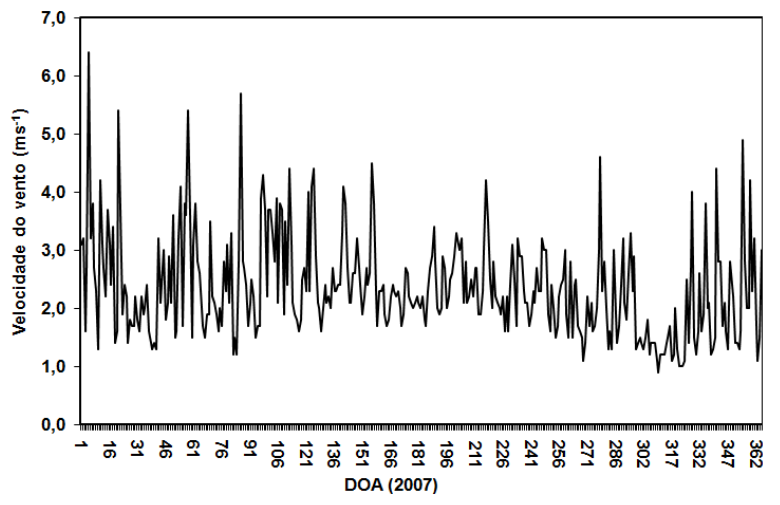

Nagler et al. (2005) encontraram que a ETr média diária da vegetação tamarisk foi de $3,9 \mathrm{~mm} \mathrm{dia}^{-1}$. Os valores anuais da $E T_{0}$ para a grama, assim como, a ETr da vegetação tamarisk na área de estudo em 2007, estão apresentados na Tabela 3. A ET da grama foi de $1734,2 \mathrm{~mm} / \mathrm{ano}$, enquanto a $E T r$ da vegetação tamarisk, no ano de 2007, foi de 1096,2 mm/ano. Os resultados

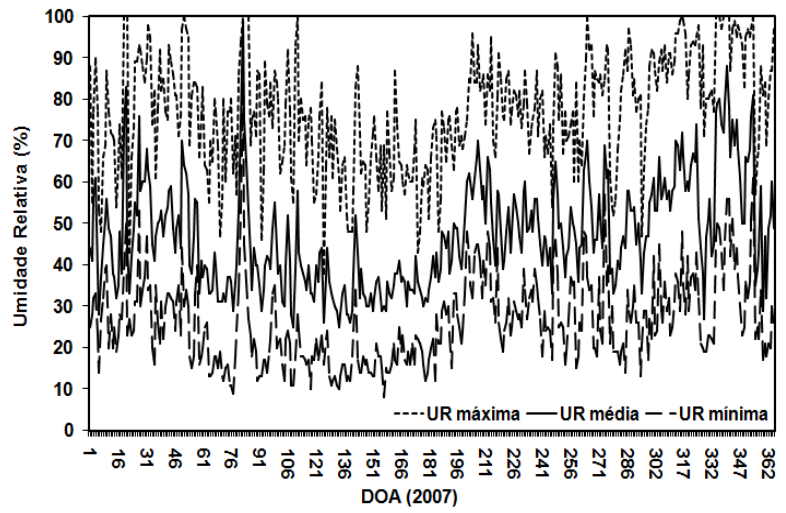

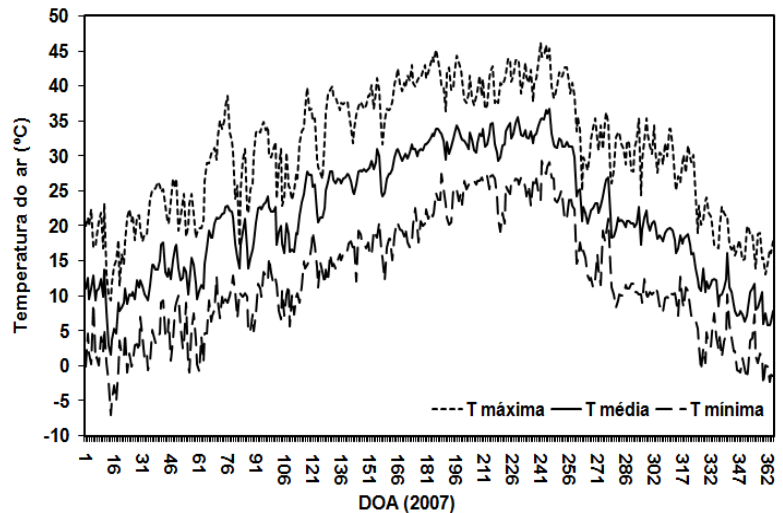

Figura 4 - Distribuição temporal da velocidade do vento (a), umidade relativa (b) e temperatura do ar (c) na estação meteorológica, durante o ano de 2007, instalada próximo da área experimental de CNWR.

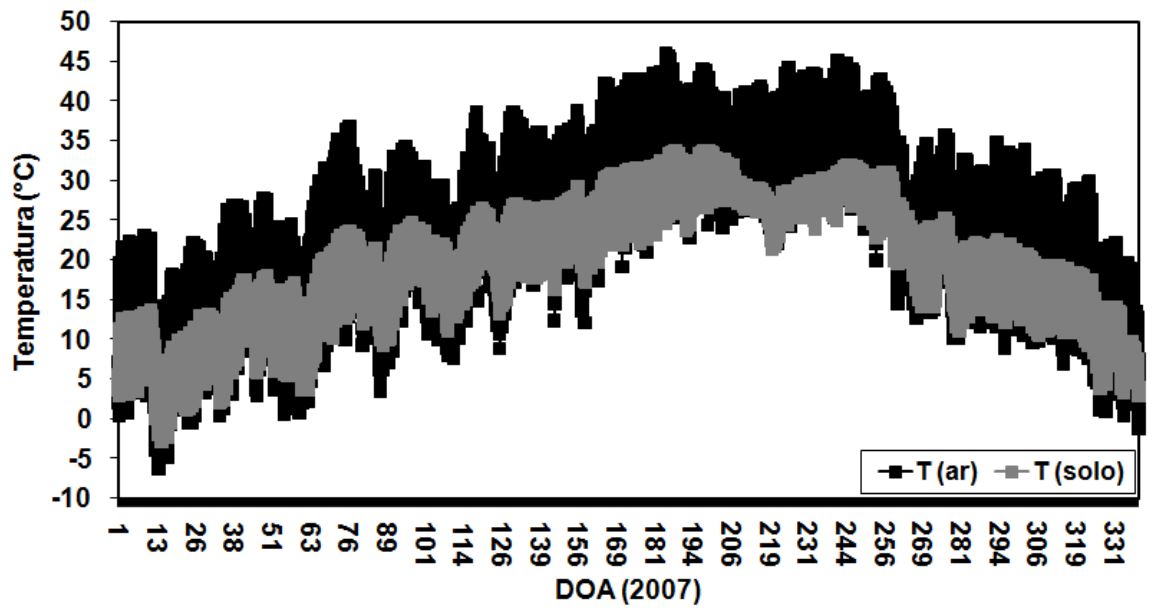

Figura 5 - Comportamento da temperatura do ar e do solo medidos na torre micrometeorológica, em CNWR no ano de 2007. 
concordam com os obtidos por Gay e Hartman (1982) e Cleverly et al. (2002).

A distribuição temporal da $E T_{0}$ (grama) e $E T r$ (tamarisk) para a área experimental no ano de 2007, está ilustrada na Figura 6. Conforme discutido anteriormente, os resultados concordam com os obtidos por Gay e Hartman (1982) e Cleverly et al. (2002) que obtiveram valores da ETr da tamarisk variando entre 0 e $12 \mathrm{mmdia}^{-1}$. A vegetação tamarisk apresentou valores médio, máximo e mínimo para a $E T r$ de 3,2 $\mathrm{mmdia}^{-1}, 7,6 \mathrm{mmdia}^{-1}$ e 0,1 mmdia $^{-1}$, respectivamente.

\subsection{Aplicação e Validação do S-SEBI na Vegetação Tamarisk}

A Tabela 4 contém os valores diários da ETr da vegetação tamarisk, medida na torre micrometeorológica com a razão de Bowen, assim como os valores estimados pelos algoritmo S-SEBI. Observa-se que, em geral, os valores estimados pelo algoritmo baseado em técnicas de sensoriamento remoto se mantiveram muito próximos dos valores medidos na torre de fluxos. Os resultados concordam com os obtidos por Nagler et al. (2005), que obtiveram valores de ETr diária, utilizando imagens MODIS para o Rio Grande no Novo México, variando entre 4,0 e 5,0 $\mathrm{mmdia}^{-1}$ para a vegetação. $\mathrm{O}$ erro quadrático médio e o erro percentual médio, entre os valores da $E T r$ diária medidos e os estimados pelo S-SEBI foi de $0,5 \mathrm{mmdia}^{-1}$ e $10,6 \%$, respectivamente. Os resultados evidenciam a aplicabilidade do método na obtenção da distribuição espacial da evapotranspiração real diária. Estes resultados estão em concordância com Weligepolage (2005), que evidencia a aplicabilidade do S-SEBI para regiões áridas e semi-áridas, assim como, onde não existem redes de estações meteorológicas bem distribuídas, uma vez que o mesmo só depende de informações da temperatura do ar no momento da passagem do satélite, o que pode ser substituído pela temperatura do píxel frio (área vegetada) (Roerink et al., 2000).

Os valores estatísticos (média, mediana e desvio padrão) da variação espacial da ETr diária, sobre toda área da CNWR, estão apresentados na Tabela 5. Observou-se que a ETr diária

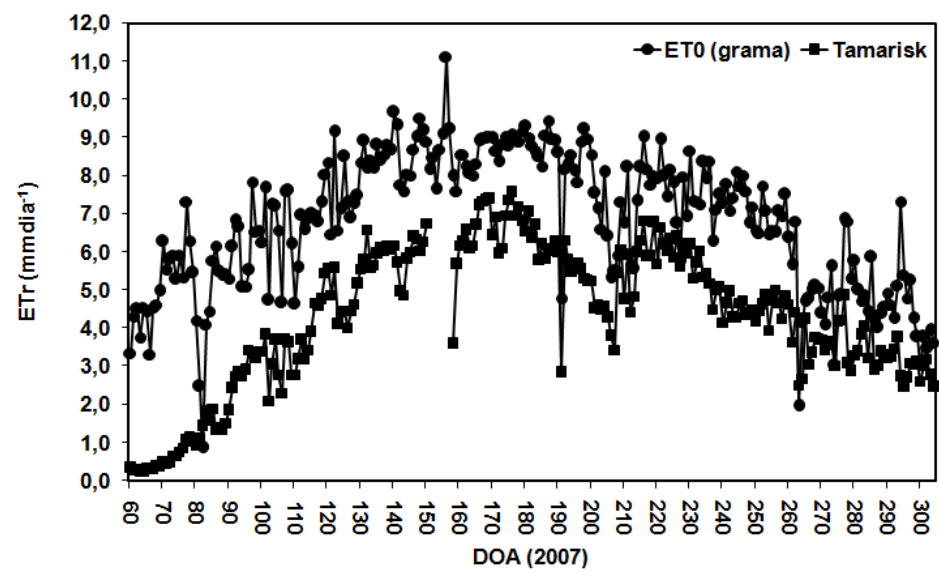

Figura 6 - Distribuição temporal da ET0 e ETr para a área experimental (CNWR, CA - EUA) para 2007.

Tabela 4 - Comparação entre a evapotranspiração real diária medida na torre micrometeorológica (razão de Bowen) e as estimadas pelo algoritmo S-SEBI, juntamente com a evapotranspiração de referência.

\begin{tabular}{|c|c|c|c|}
\hline DOA & $\mathrm{ET}_{0}\left(\mathrm{~mm} \mathrm{dia}{ }^{-1}\right)$ & $\operatorname{ETr}($ medido $)\left(\mathrm{mm} \mathrm{dia}^{-1}\right)$ & $\operatorname{ETr}(\mathrm{S}-\mathrm{SEBI})\left(\mathrm{mm} \mathrm{dia}^{-1}\right)$ \\
\hline 128 & 6,9 & 4,6 & 5,5 \\
\hline 144 & 7,7 & 5,8 & 7,6 \\
\hline 160 & 7,9 & 6,2 & 6,0 \\
\hline 192 & 7,8 & 6,3 & 5,8 \\
\hline 224 & 7,5 & 6,4 & 5,4 \\
\hline 240 & 6,8 & 4,1 & 4,8 \\
\hline 256 & 6,0 & 5,0 & 4,5 \\
\hline
\end{tabular}


Tabela 5 - Estatísticas da variação espacial da ETr diária da vegetação tamarisk obtida pelo algoritmo S-SEBI, sobre o CNWR, no ano de 2007

\begin{tabular}{cccc}
\hline \multirow{2}{*}{ DOA } & \multicolumn{3}{c}{ ETr S-SEBI $\left(\mathbf{m m ~ d i a}^{-1}\right)$} \\
\cline { 2 - 4 } & Média & Mediana & Desvio padrão \\
\hline 128 & 4,0 & 4,0 & 1,0 \\
144 & 5,5 & 5,6 & 1,3 \\
160 & 4,6 & 4,7 & 1,1 \\
192 & 4,7 & 4,9 & 0,9 \\
224 & 4,3 & 4,4 & 0,9 \\
240 & 3,9 & 4,0 & 0,9 \\
256 & 3,6 & 3,7 & 0,8 \\
\hline
\end{tabular}
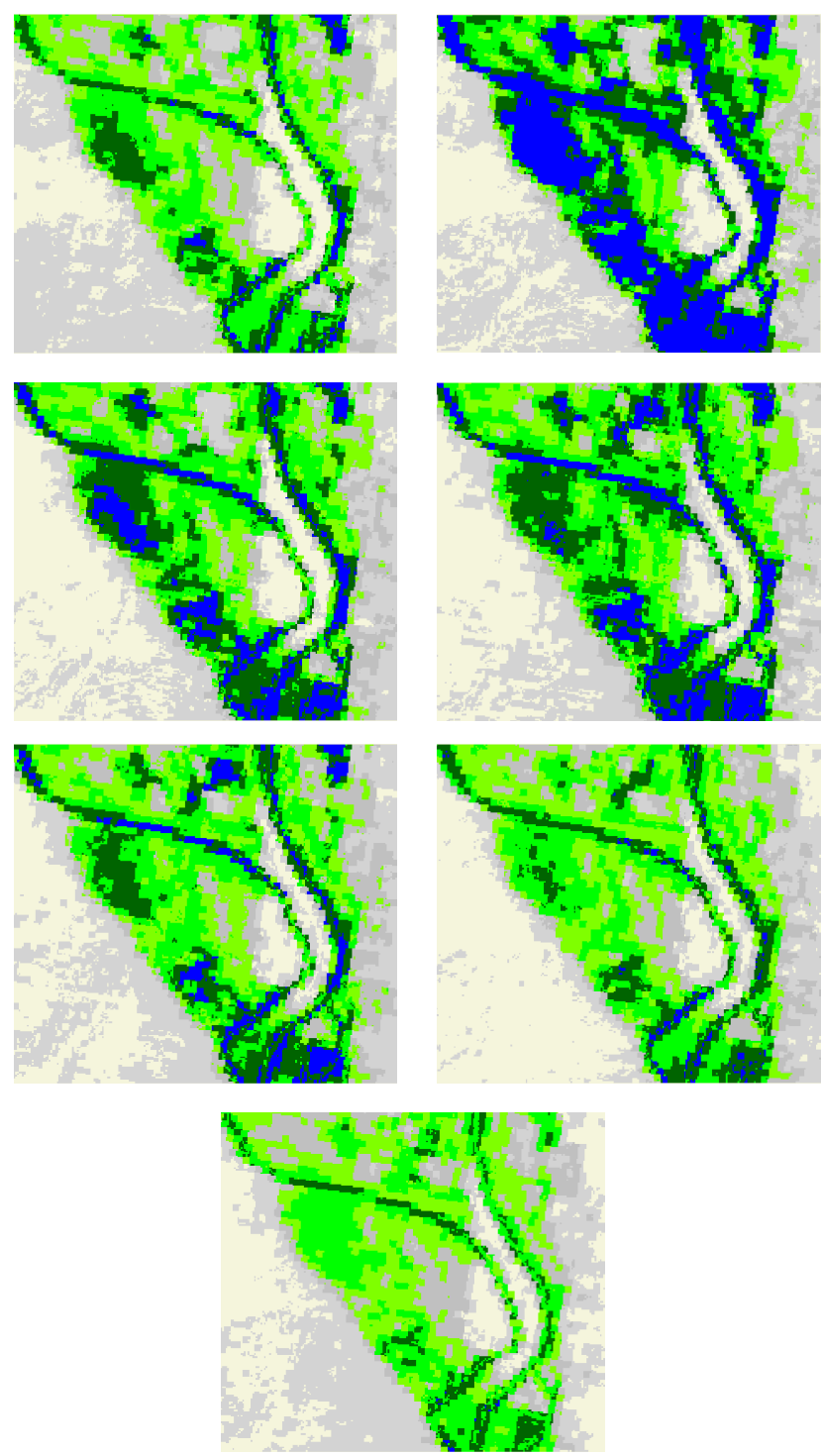

Figura 7 - Distribuição espacial da ETr para toda área da CNWR nos DOA 128(a), 144(b), 160(c), 192(d), 224(e), 240(f) e 256(g) obtida pelo algoritmo S-SEBI. foi superior aos $3,5 \mathrm{~mm} \mathrm{dia}^{-1}$, evidenciando o alto consumo hídrico da vegetação tamarisk. O desvio padrão alcançou valores variando entre $0,7 \mathrm{e} 1,4 \mathrm{~mm} \mathrm{dia}^{-1}$, o que caracteriza as diferentes densidades de vegetação dentro da CNWR.

Nas Figuras $7 \mathrm{a}-7 \mathrm{~g}$ estão mostradas as distribuições espaciais da ETr diária, obtida pelo S-SEBI, para toda a área estudada, nos diferentes dias do ano. É possível identificar nas imagens as áreas com diferentes densidades de vegetação tamarisk. As áreas em azul e verde escuras apresentam valores de $E T r$ iguais ou superiores a $5 \mathrm{~mm} \mathrm{dia}^{-1}$, o que representa vegetação tamarisk densa. As áreas em cinza escuro e verde claras têm $E T r$ variando entre 2 e $5 \mathrm{~mm} \mathrm{dia}^{-1}$, representando as áreas de vegetação de médio porte, e as áreas em cinza claro representam vagetação de baixo porte e esparsa e/ou solo exposto, a exemplo de áreas de queimadas e deserto, com ETr inferior a $2 \mathrm{~mm} \mathrm{dia}^{-1}$.

\section{CONCLUSÕES}

Foi evidenciado através da velocidade do vento, características de forte advecção de ar sobre a região estudada, e que a maior variação na velocidade média diária do vento ocorre no período mais frio do ano. No clima desértico da região estudada, a temperatura do ar é elevada durante o dia e baixa à noite.

Foi possível identificar que a $E T r$ da vegetação tamarisk apresentou valores concordantes com os estudos desenvolvidos por Nagler et al. (2005) e Nagler et al. (2008) para a região estudada utilizando sensoriamento remoto e torres micrometeorológicas. As estimativas da ETr pelo algoritmo S-SEBI são similares aos valores medidos na torre micrometeorológica. Os baixos erros quadrático médio e percentual médio entre os valores da ETr diária medidos e os estimados pelo S-SEBI, assim como, a identificação da dinâmica da vegetação através da distribuição espacial da evapotranspiração, evidenciam a aplicabilidade do método na obtenção da evapotranspiração real diária em regiões áridas e semi-áridas.

\section{AGRADECIMENTOS}

Ao $\mathrm{CNPq}$ (Conselho Nacional para o Desenvolvimento Científico e Tecnológico), pela bolsa de doutorado concedida ao primeiro autor. Ao prof. Dr. Christopher M. U. Neale (BIE/ Utah State University, EUA) pelo acolhimento e apoio durante todo o período de estágio no exterior. Aos Drs. John Osterberg (U.S. Bureau of Reclamation, Denver, CO), Doyle Watts (Wright State University) e Subramania I. Sritharan (Central State University) pela liberação dos dados micrometeorológicos da vegetação tamarisk. 


\section{REFERÊNCIAS BIBLIOGRÁFICAS}

ALLEN, R. G.; PEREIRA, L. S.; RAES, D.; SMITH, M. Crop evapotranspiration - Guidelines for computing crop water requirements - FAO Irrigation and drainage paper 56. Rome-Italy, 1998.

BASTIAANSSEN, W. G. M.; MENENTI, M.; FEDDES, R. A.; HOLTSlAG, A. A. M. A remote sensing surface energy balance algorithm for land (SEBAL) 1. Formulation. Journal of Hydrology, v. 212-213, p.198-212. 1998a.

BASTIAANSSEN, W. G. M.; PELGRUM, H.; WANG, J.; MA, Y.; MORENO, J. F. ROENRINK, G. J. VAN DER WAL, T. A remote sensing surface energy balance algorithm for land (SEBAL) 2. Validation. Journal of Hydrology, v. 212-213, p.213-229. 1998b.

BASTIAANSSEN, W. G. M. SEBAL-based sensible and latent heat fluxes in the irrigated Gediz Basin, Turkey. Journal of Hidrology, v. 229, p. 87-100, 2000.

BOWEN, I. S. The ratio of heat losses by conduction and by evaporation from any water surface. Physical Review, v. 27, p. 779-787, 1926.

CleVERLY, J. R.; DAHM, C. N.; THIBAULT, J. R.; GILROY, D. J.; COONROD, J. E. A. Seasonal estimates of actual evapotranspiration from Tamarix ramossissima stands using three-dimensional eddy covariance. Journal of Arid Environments, v. 52, p. 181-197, 2002.

GAY, L.W.; HARTMAN, R.K. ET measurements over riparian saltcedar on the Colorado River. Hydrology and Water Resources in Arizona and the Southwest, v.12, p.133-139, 1982.

GÓMEZ, M.; OLIOSO, A.; SOBRINO, J. A.; JACOB, F. Retrieval of evapotranspiration over the Alpilles/ReSeDA experimental site using airborne POLDER sensor and thermal camera. Remote Sensing of Environments, v. 96, p. 399-408, 2005.

HEILMAN, J. L.; BRITTIN, C. L.; NEALE, C. M. U. Fetch requirements for Bowen ratio measurements of latent and sensible heat fluxes. Agricultural and Forest Meteorology, v.44, p. 261-273, 1989.

MACK, R. N.; SIMBERLOFF, D.; LONSDALE, W. M.; EVANS, H.; CLOUT, M.; BAZZAZ, F. A. Biotic invasions: causes, epidemiology, global consequences, and control. Ecological Applications, v. 10, p. 689-710, 2000 .

NAGLER, P. L.; SCOTT, R. L.; WESTENBURG, C.; CleVERLY, J. R.; GLENN, E. P.; HuETE, A. R. Evapotranspiration on western U.S. rivers estimated using the Enhanced Vegetation Index from MODIS and data from eddy covariance and Bowen ratio flux towers.
Remote Sensing of Environment, v. 97, p. 337-351, 2005.

NAGLER, P. L.; GLENN, E. P.; DIDAN, K.; OSTERBERG, J.; JORDAN, F.; CUNNINGHAM, J. Wide-Area Estimates of Stand Structure and Water Use of Tamarix spp. On the Lower Colorado River: Implications for Restoration and Water Management Projects. Restoration Ecology, v. 16, p. 136-145, 2008.

PAYERO, J. O.; NEALE, C. M. U.; WRIGHT, J. L.; ALLEN, R. G. Guidelines For Validating Bowen Ratio Data. Transactions of the ASAE, v. 46, p. 1051-1060, 2003.

PEREZ, P. J.; CASTELLVI, F.; MARTÍNEZ-COB, A. A simple model for estimating the Bowen ratio from climatic factors for determining latent and sensible heat fluxes. Agricultural and Forest Meteorology, v. 148, p. 25-37, 2008.

PIMENTEL, D.; MCNAIR, S.; JANECKA, J.; WIGHTMAN, J.; SIMMONDS, C.; O'CONNELL, C.; WONG, E.; RUSSEL, L.; ZERN, J.; AQUINO, T.; TSOMONDO, T. Economic and environmental threats of alien plant, animal, and microbe invasions. Agriculture, Ecosystems and Environment, v. 84, p. 1-20, 2001.

ROERINK, G. J.; SU, Z.; MENENTI, M. A Simple Remote Sensing Algorithm to Estimate the Surface Energy Balance. Physics and Chemistry of the Earth (B), v. 25, p. 147$157,2000$.

SANTOS, C.A. C.; SILVA, B. B. Estimativa da evapotranspiração da bananeira em região semi-árida através do algoritmo S-SEBI. Revista Brasileira de Agrometeorologia, v. 16, p. 9-20, 2008.

SHAFROTH, P. B.; CLEVERLY, J. R.; DUDLEY, T. L.; TAYLOR, J. P.; VAN RIPPER III, C.; WEEKS, E. P.; STUART, J. N. Control of Tamarix in the Western United States: Implications for Water Salvage, Wildlife Use, and Riparian Restoration. Environmental Management, v. 35, p. 231-246, 2005.

SOBRINO, J. A.; GÓMEZ, M.; JIMÉNEZ-MUÑOZ, J. C.; OLIOSO, A.; CHEHBOUNI, G. A simple algorithm to estimate evapotranspiration from DAIS data: Application to the DAISEX Campaigns. Journal of Hydrology, v. 315, p. 117-125, 2005.

SOBRINO, J. A.; GÓMEZ, M.; JIMÉNEZ-MUÑOZ, J. C.; OLIOSO, A. Application of a simple algorithm to estimate daily evapotranspiration from NOAA-AVHRR images for the Iberian Peninsula. Remote Sensing of Environment, v. 110, p. 139-148, 2007.

SWINBANK, W. C. The measurement of vertical transfer of heat and water vapor by eddies in the lower atmosphere. Journal of Meteorology, v. 8, p. 135-145, 1951.

TANNER, B. D. Use requirement for Bowen ratio and eddy correlation determination of evapotranspiration. In Proc. 
ASCE 1988 Specialty Conference of the Irrigation and Drainage Division of the ASCE, 605-616. Reston, Va.: ASCE. 1988.

TANNER, B. D.; GREENE, J. P.; BINGHAM, G. E. A Bowen ratio design for long term measurements. In Proc. ASAE 1987 International Winter Meeting. St. Joseph, Mich.: ASAE, 1987.

TREZZA, R. Evapotranspiration using a satellite-based Surface energy balance with standardized ground control. $247 \mathrm{f}$. Thesis (Doctor of Philosophy in Biological and Agricultural Engineering). Utah State University. Logan, Utah, 2002.

VAN RIPPER III, C.; PAXTON, K.; O'BRIEN, C.; SHAFROTH, P.; MCGRATH, L. Rethinking avian response to Tamarisk on the Lower Colorado River: a threshold hypothesis. Restoration Ecology, v. 16, p. 155-167, 2008.
VERMA, S. B.; ROSENBERG, N. J.; BLAD, B. L. Turbulent exchange coefficients for sensible heat and water vapor under advective conditions. Journal of Applied Meteorology, v. 17, p. 330-338, 1978.

WEEKS, E. P.; WEAVER, H. L.; CAMPBELL, G. S.; TANNER, B. D. Water use by saltcedar and by replacement vegetation in the Pecos River floodplain between Acme and Artesia, New Mexico. US Geological Survey Professional Paper, 491-G, 33 p., 1987.

WELIGEPOLAGE, K. Estimation of spatial and temporal distribution of evapotranspiration by satellite remote sensing -A case study in Hupselse Beek, The Netherlands. 2005, 114 p, Dissertation (Master of Science), International Institute for Geo-information Science and Earth Observation, Waganingen, Netherlands, 2005. 